\title{
Non-Markov excursion set model of dark matter halo abundances ${ }^{\star}$
}

\author{
G. Amosov ${ }^{1}$ and P. Schuecker ${ }^{2}$ \\ 1 Moscow Institute of Physics and Technology, Institutski 9, 141700 Dolgoprudni, Russia \\ 2 Max-Planck-Institut für extraterrestrische Physik, Giessenbachstraße, 85741 Garching, Germany \\ e-mail: peters@mpe.mpg.de
}

Received 2 October 2003 / Accepted 11 February 2004

\begin{abstract}
The excursion set model provides a convenient theoretical framework to derive dark matter halo abundances. This paper generalizes the model by introducing a more realistic merging and collapse process. A new parameter regulates the influence of the environment and thus the coherence (non-Markovianity) of the merging and the collapse of individual mass shells. The model mass function also includes the effects of an ellipsoidal collapse. Analytic approximations of the halo mass function are derived for scale-invariant power spectra with the slopes $n=0,-1,-2$. The $n=-2$ mass function can be compared with the results obtained from the "Hubble volume" simulations. A significant detection of non-Markovian effects is found for an assumed accuracy of the simulated mass function of $10 \%$.
\end{abstract}

Key words. galaxies: clusters: general - cosmology: theory - cosmology: dark matter

\section{Introduction}

The hierarchical growth of virialized cosmic structures provides a useful physical paradigm for the understanding of the formation of galaxies and clusters of galaxies in the Universe (White \& Rees 1978). The Zel'dovich (1970) theory also includes a description of partially virialized structures like filaments and walls. In both models, cosmic structures grow from initial Gaussian density fluctuations via gravitational instability, leading after merging and their (partial) virialization to mass functions which are used as powerful statistical diagnostics.

Theoretical mass functions of dark matter halos are estimated from $N$-body simulations with accuracies of $10-30 \%$ (e.g. Jenkins et al. 2001). More physical insights can be obtained from analytic treatments based on Press-Schechter (PS)-like arguments (Press \& Schechter 1974; Bond et al. 1991, hereafter BCEK). Alternative derivations are based on, e.g., non-Gaussian statistics (Lucchin \& Matarrrese 1988), or treat both fluctuations and interactions of density perturbations within one process (Cavaliere \& Menci 1994), or directly consider the non-linear regime (Valageas \& Schaeffer 1997). The inclusion of non-spherical dynamical approximations (Monaco 1995; Lee \& Shandarin 1998) and ellipsoidal collapse models (Sheth et al. 2001, hereafter SMT, 2002) modify the assumption of a spherical collapse while preserving the simplicity of the original excursion set idea.

\footnotetext{
Send offprint requests to: G. Amosov, e-mail: amosov@fizteh.ru

* Appendix A is only available in electronic form at http://www. edpsciences.org
}

The excursion set model of BCEK assumes a Gaussian density field which is smoothed at a given spatial location with a top-hat filter in wavenumber space (sharp $k$-space filter) using different comoving filter radii $R$. The resulting filtered density contrasts $\delta(R)$ perform a highly jagged diffusion trajectory (Fig. 1) where the mass function is derived - as a function of the standard deviation $\sigma(R)$ of the mass density fluctuations from the loss rate of trajectories at the barrier $\delta_{\mathrm{c}}$ at their lowest $\sigma$ level (highest $R$ or mass scale). For the spherical collapse model and for the Einstein-de Sitter Universe we have $\delta_{\mathrm{c}}=1.686$, weakly dependent on cosmology. The halo mass function is thus directly related to the first passage time distribution of the trajectories.

There is a problem with this approach related to the assumption of a sharp $k$-space filter. The filter gives a quite unrealistic mass assignment scheme, with a growth of cosmic structure which depends only on the mass of a halo it has within an infinitesimally small time interval at a given cosmic epoch, and without any dependency on past or future properties of the halo (Markov assumption). Therefore, the merging events occur as completely uncorrelated, sudden, jumps in the formation history. We will replace the sharp $k$-space filter by a non-sharp filter. This leads to a more realistic mass assignment scheme. The corresponding growth of cosmic structure depends on the properties of the halo over a finite and future-directed time range (non-Markov assumption).

The idea to invoke non-Markovian processes is not new. A discussion of the physical consequences of the Markov assumption and why related processes could fail can be found in White $(1996,1997)$. BCEK pointed out the relation between 


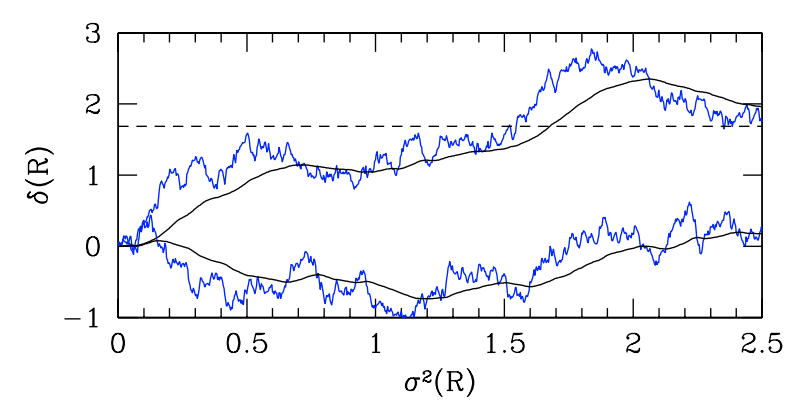

Fig. 1. Trajectories for the extended PS process (Markovian process, jagged curves, $T=0$ ) and for the non-Markovian process (smooth curves, $T=0.23$ ).

the shape of a mass filter and the Markov assumption. Their discussion of non-Markovian processes is, however, mainly restricted to results obtained with Monte-Carlo experiments.

Using the traditional excursion set model as a guideline, Sect. 2 introduces a simple analytic model which describes a more uniform mass assignment scheme, and thus a more uniform spherical collapse of dark matter halos. This model is further improved in Sect. 3, by including the effects of an ellipsodial collapse similar to SMT. The combined model can be regarded as a simple though typical example of a non-Markovian process. It generalizes the traditional excursion set model in a manner such that non-Markovianity can now be gradually increased by a new filter parameter. The same filter parameter also increases the smoothness of the profile of the mass filter (see Eq. (8) in Sect. 4).

The standard mirror image method is used in Sect. 5 to derive an analytic form for the halo mass function. We show why this method, which in general does not work for nonMarkovian processes, can be used in our specific case. The resulting mass function has the same functional form as the standard excursion set result in terms of the variance of the mass distribution and the critical density threshold. However, non-Markovian effects change the relations between filter radius, mass and variance. These relations become a function of the power spectrum of the underlying mass distribution. After the corresponding transformations of the mass functions they also become apparent in the halo mass function itself. This is the reason why in our non-Markovian context, the profile of the mass filter and thus how much mass is swep in from the surrounding mass of a collapsing region becomes a function of the power spectrum.

How much mass the filter sweeps in for a given filter radius can be a quite complex function, especially when general power spectra are considered. We could derive approximate analytic results for scale-invariant power spectra with the slopes $n=0,-1,-2$. The latter case is close to the observed value and allows a comparison with the Jenkins et al. (2001) mass function obtained from the "Hubble Volume" simulations (Sect. 6). The basic aim is to test under the given assumptions the presence of non-Markovian effects in the simulations and to determine how accurately mass functions should be measured to detect the effects. A discussion of general power spectra goes beyond the scope of our analytic treatment and is postponed to a further paper.

\section{Spherical collapse and merger trajectories}

The Markov assumption corresponds to an oscillating filter in configuration space or a sharp filter in $k$-space. For the determination of the total collapsed mass of a halo, the formal weighting of each mass shell with an oscillating filter suggests the following collapse picture. The innermost mass shell located around a peak in the cosmic mass distribution (though not necessarily around a massive particle) is up-weighted in the mass budget and is thus expected to collapse, whereas the next overlaying mass shell located around the first minimum of the filter is down-weighted, the following mass shell is up-weighted again, and so on. Similarily, for the determination of merger trajectories, the sharpness of the filter in $k$-space leads to the addition of statistically independent fluctuation power in disjunct $k$-shells and thus to highly jagged trajectories with completely independent increments. Both the collapse and the merger trajectories are regard as unrealistic (Fig. 1).

BCEK showed that a smooth, non-oscillating filter can be obtained with a non-Markovian process. It is also well-known that smooth filters like a Gaussian or a standard top-hat are consistent with the uniform (coherent) collapse of all concentric mass shells. Moreover, the smoothness of the filter in $k$-space mixes fluctuation power located in disjunct $k$-shells and thus smoothes the merger trajectories. We are thus searching for diffusion processes with smooth merger trajectories. The physical significance of the corresponding mass filter is evaluated by the comparison with mass functions derived from simulations (Sect. 6).

Formally, the merger trajectories of the excursion set model are described by the Wiener stochastic diffusion process $W(t)-$ the Brownian motion. We follow the general convention and work with a pseudo time or mass resolution variable $t(R)$ in $\sigma(t)$ which will be specified later (Eq. (9)), and the filtered density contrast $\delta(\sigma)$ (see BCEK and Lacey \& Coles 1993 for more details). A non-Markovian process with the desired properties has the increments of the density contrast

$\mathrm{d} \delta(t)=\left[\frac{1}{T} \int_{0}^{t} \mathrm{e}^{-\frac{t-s}{T}} \mathrm{~d} W(s)\right] \mathrm{d} t$.

The increments depend on all values of $W(t)$ within $[0, t]$, and if the parameter $T$ tends to zero, the smoothness of the merger trajectories diminishes (Fig. 1). Whereas the sum over history is in most cases a generic feature of non-Markovian processes, the kernel function in Eq. (1) may not necessarily be exponential. However, its explicit form comes from the shape of the mass filter function (Eq. (8)) and finds its justification through the usability of this mass filter. Its shape suggests more realistic extraction of the material which will ultimately end up in a spherical collapse to form a virialized halo. The new parameter $T$ measures the coherence (non-Markovianity) of the collapsing mass shells and can be estimated from fits of halo mass functions to simulated or observed data (Sect. 6).

\section{Ellipsoidal collapse and non-Markovianity}

The theory of ellipsoidal collapse (Bond \& Myers 1996, SMT) provides a relation between the critical density contrast of an 
ellipsoidal collapse $\delta_{\mathrm{ec}}$ and a spherical collapse $\delta_{\mathrm{c}}$ of the form $\delta_{\mathrm{ec}}=\delta_{\mathrm{c}}+\beta \delta_{\mathrm{c}}\left(\sigma / \delta_{\mathrm{c}}\right)^{2 \gamma}$. For convenience we set $\gamma=1$, yielding mathematically exact first passage time distributions. Our approximation

$\delta_{\mathrm{ec}}=\delta_{\mathrm{c}}+\frac{\beta}{\delta_{\mathrm{c}}} \sigma^{2}$

deviates from the exact result on the $10 \%$ level or better for $\beta=0.3$ within $0.0<\sigma^{2}<1.5$. In the diffusion model of merger trajectories, the ellipsoidal collapse (Eq. (2)) induces a linear drift superposed onto the diffusion process (Eq. (1)) where high (low) mass objects needs low (high) effective critical density contrasts for their virialization. We thus rewrite Eq. (1) as

$$
\begin{aligned}
\mathrm{d} \delta(t) & =\left[\frac{1}{T} \int_{0}^{t} \mathrm{e}^{-\frac{t-s}{T}} \mathrm{~d} W(s)\right] \mathrm{d} t-\beta \mathrm{d} \sigma^{2}(t) \\
& =\frac{1}{T}\{W(t)-[\delta(t)-\mathrm{E}(\delta(\mathrm{t}))]\} \mathrm{d} t-\beta \frac{\mathrm{d}}{\mathrm{d} t} \operatorname{Var}(\delta(t)) \mathrm{d} t, \\
\delta(0) & =0,
\end{aligned}
$$

where $T$ and $\beta$ are fixed positive numbers. The solution of Eq. (3) can be represented as

$\delta(t)=\int_{0}^{t}\left(1-\mathrm{e}^{-\frac{t-s}{T}}\right) \mathrm{d} W(s)-\beta \sigma^{2}(t)$,

with the expectation $\mathrm{E}(\delta(t))=-\beta \sigma^{2}(t)$ and the variance

$$
\begin{aligned}
\sigma^{2}(t) & =\operatorname{Var}(\delta(t))=\int_{0}^{t}\left(1-\mathrm{e}^{-\frac{t-s}{T}}\right)^{2} \mathrm{~d} s \\
& =t-\frac{3}{2} T+2 T \mathrm{e}^{-\frac{t}{T}}-\frac{T}{2} \mathrm{e}^{-\frac{2 t}{T}} .
\end{aligned}
$$

For some applications (Eqs. (12), (14), (16)) it is convenient to have the inverse of Eq. (5). A good approximation is

$t\left(\sigma^{2}\right)=\sigma^{2}(t)+1.98 T\left\{1-\mathrm{e}^{-\left[\frac{\sigma^{2}(t)}{T}\right]^{0.363}}\right\}$,

which has an accuracy of better than $2 \%$ for $\sigma \leq 1$ and $0 \leq$ $T<5$.

The smoothness (differentiability) of the sample trajectories (Fig. 1) is a clear signature of the non-Markovianity of the process (Eq. (4)). A general discussion of non-Markovianity can be found in Feller (1968). As mentioned above, a nonMarkovian process can be obtained by integrating certain Markov processes. It is well-known from diffusion theory that if one starts with the Markov process $V(t)$ representing particle velocities in a viscid medium, then positions $X(t)$ defined as $\mathrm{d} X(t)=V(t) \mathrm{d} t$ form the simplest non-Markovian process. Note that setting a complete path $X(s), t-T \leq s \leq t$, is not the same as setting only one position $X(t)$ which provides the nonMarkov property of $X(t)$. In our case, the process $\delta(t)$ can be represented as an integral over the Ornstein-Uhlenbeck process (see Sect. 5) and is thus non-Markovian. Moreover, the $\delta(t)$ are Gaussian and have a probability distribution which is similar to the probability distribution of $W(t)-\beta t$.

\section{Filter function}

The mass filter of the non-Markovian process can be derived as in Schuecker et al. (2001a) by equating the process variance (Eq. (5)) and the variance of the underlying matter fluctuations,

$\int_{0}^{t} \mathrm{~d} s\left(1-\mathrm{e}^{\frac{s-t}{T}}\right)^{2}=\int_{0}^{\infty} \frac{4 \pi k^{2} \mathrm{~d} k P(k)}{(2 \pi)^{3}}\left|W_{T}(k R)\right|^{2}$,

where $P(k)$ is the matter power spectrum, $W_{T}(k R)$ the mass filter function in $k$-space, and $R$ the filter scale in configuration space. Equation (7) cannot be solved easily for an arbitrary $P(k)$. However, for scale-invariant power spectra of the form $P(k)=P_{0} k^{n}$ the filter

$W_{T}(k R)=1-\exp \left\{\frac{P_{0}}{2 \pi^{2}(n+3) R^{n+3}}\left[\frac{(k R)^{n+3}-1}{T}\right]\right\}$,

for $k R \leq 1$, and $W_{T}(k R)=0$ elsewhere, is consistent with Eq. (5) when the resolution variable is defined as

$t=\frac{P_{0}}{2 \pi^{2}(n+3) R^{n+3}}$.

Here, $t$ is the same as in the Markov excursion set model and requires $n>-3$ for structure to grow through hierarchical clustering.

A new aspect is the dependence (for $T>0$ ) of the filter on the power spectrum (Fig. 2). For standard filters, the profile does not change with $P(k)$ and only normalization factors like the characteristic mass scale $M_{*}$ used to get convenient invariance properties of the halo mass function depend on $P(k)$. In the present case the filter profile depends on $P(k)$ and thus on the spatial correlation function. The correlation function approximates the mean shape of density peaks. Therefore, the profile of the filter roughly follows the mean profile of density peaks.

\subsection{Filter volumes}

From the volume $V_{T}$ of the filter we can estimate the mass $M=\bar{\rho} V_{T}$ extracted by the filter from the mean cosmic matter density $\bar{\rho}$. The volume $V_{T}(R)$ is related to the Fourier transform of Eq. (8) by

$\frac{1}{V_{T}(R)}=W_{T}(r=0, R)=\frac{1}{2 \pi^{2}} \int_{0}^{1 / R} \mathrm{~d} k k^{2} W_{T}(k R)$.

For the filter (Eq. (8)), three cases can be studied in a straightforward manner. For a power spectrum with the spectral in$\operatorname{dex} n=0$ we have

$\frac{1}{V_{T}}=\frac{1}{6 \pi^{2} R^{3}}-\frac{T}{P_{0}}\left(1-\mathrm{e}^{-\frac{P_{0}}{6 \pi^{2} T R^{3}}}\right)$,

$P_{0}=3072 \pi^{2}\left[\sigma_{8}^{2}+1.98 T\left(1-\mathrm{e}^{-\left(\frac{\sigma_{8}^{2}}{T}\right)^{0.363}}\right)\right] \mathrm{Mpc}^{3}$.

For $n=-1$ integration of Eq. (10) yields

$$
\begin{aligned}
\frac{1}{V_{T}}= & \frac{1}{6 \pi^{2} R^{3}}-\frac{T}{P_{0} R}+\left(\frac{\pi T}{P_{0}}\right)^{3 / 2} \\
& \times \mathrm{e}^{-\frac{P_{0}}{4 \pi^{2} R^{2} T}} \operatorname{erfi}\left(\frac{1}{2 \pi R} \sqrt{\frac{P_{0}}{T}}\right),
\end{aligned}
$$



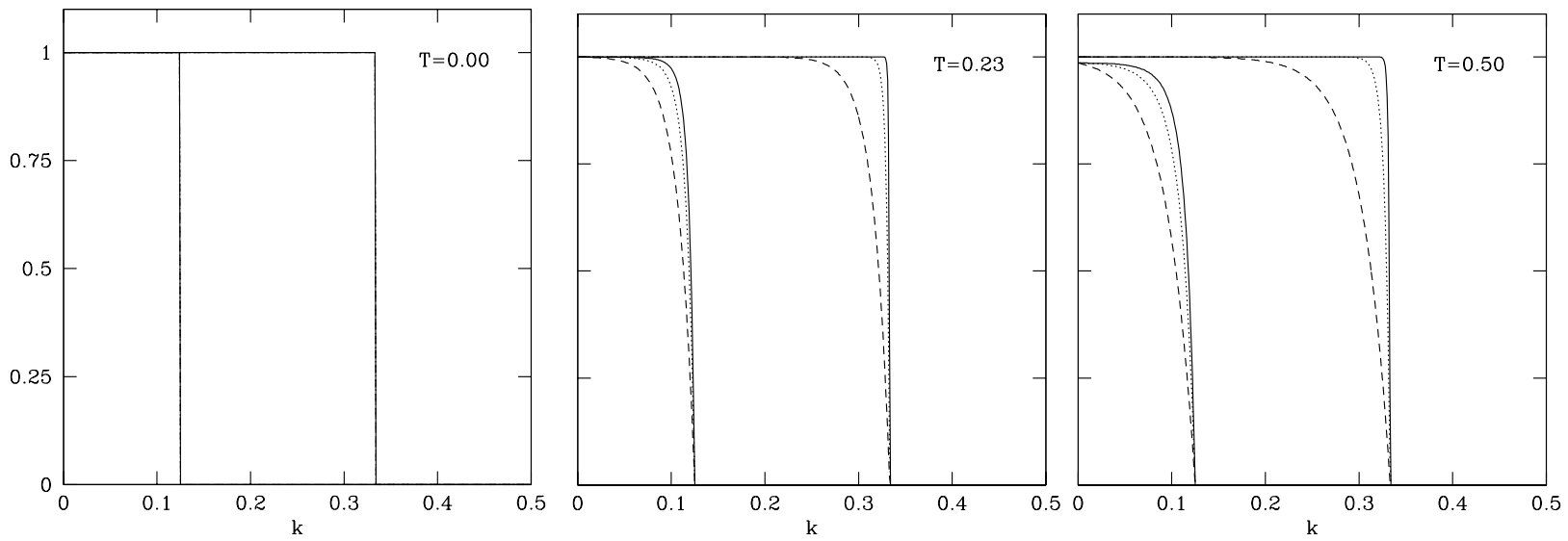

Fig. 2. Filter profiles $W_{T}(k)$ as a function of comoving wavenumber $k$ in units of $\left[h \mathrm{Mpc}^{-1}\right.$ ] for three values of the $T$ parameter $(l e f t: T=0.00$, middle: $T=0.23$, right: $T=0.50$ ). Each panel shows two groups of filters. Left group: filter radius $R=8 h^{-1} \mathrm{Mpc}$, right group: $R=3 h^{-1} \mathrm{Mpc}$. Each group consists of three filter curves. From up to down: $n=0$ (continuous), -1 (dotted) ,-2 (dashed). The normalization of the power spectrum $P_{0}$ is adjusted to give $\sigma_{8}=0.9$ (at $T=0$ ).

$P_{0}=256 \pi^{2}\left[\sigma_{8}^{2}+1.98 T\left(1-\mathrm{e}^{-\left(\frac{\sigma_{8}^{2}}{T}\right)^{0.363}}\right)\right] \mathrm{Mpc}^{2}$.

For $n=-2$ integration of Eq. (10) yields

$\frac{1}{V_{T}}=\frac{1}{6 \pi^{2} R^{3}}-\frac{T}{P_{0} R^{2}}+\frac{4 \pi^{2} T^{2}}{P_{0}^{2} R}-\frac{8 \pi^{4} T^{3}}{P_{0}^{3}}\left(1-\mathrm{e}^{-\frac{P_{0}}{2 \pi^{2} R T}}\right)$,

$P_{0}=16 \pi^{2}\left[\sigma_{8}^{2}+1.98 T\left(1-\mathrm{e}^{-\left(\frac{\sigma_{8}^{2}}{T}\right)^{0.363}}\right)\right] \mathrm{Mpc}$,

where Eqs. (12), (14), (16) use the approximation (Eq. (6)), and $\sigma_{8}$ is a parameter determined by the filter (Eq. (8)) analogous to the standard normalization of the power spectrum. In Eq. (13), erfi is the imaginary error function. In all three cases, for a given radius the volume and thus the mass covered by a filter with $T>0$ is larger compared to $T=0$ where we have $V_{0}=6 \pi^{2} R^{3}$.

\subsection{Filter radius-mass relations}

For the mass function, the relation between the filter radius and mass are needed. Therefore, Eqs. (11), (13), (15) have to be solved for $R$. For $n=0$ we have

$$
\begin{aligned}
& R(M)=\frac{M^{1 / 3}}{\left\{6 \pi^{2} \bar{\rho}+\frac{6 \pi^{2} T M}{P_{0}}[1+W(z)]\right\}^{1 / 3}}, \\
& z=-\frac{1}{\exp \left(1+\frac{\bar{\rho} P_{0}}{M T}\right)},
\end{aligned}
$$

with $W(z)$ the principal branch of the Lambert W-function (e.g. Corless et al. 1996, not to be confused with the Wiener process in Eq. (3)). In the present case we have $\infty>T \geq 0$ which corresponds to the range $-1 / e<z \leq 0$ where $W(z)$ is monotonically increasing with values in the interval $-1<W(z) \leq 0$. In some cases (Eq. (23)) the derivative $\mathrm{d} W(z) / \mathrm{d} z=W /[z(1+W)]$ is needed.
For $n=-1$ we can neglect the final exponential term in Eq. (13) which has an effect of $<5 \%$ for $T \leq 1$ up to halo masses of $10^{16} M_{\odot}$. Solving the truncated equation for $R$ yields

$R(M)=\frac{\left(U_{1}+3 \sqrt{U_{2}}\right)^{1 / 3}}{U_{3}}+\frac{U_{4}}{\left(U_{1}+3 \sqrt{U_{2}}\right)^{1 / 3}}-U_{5}$,

where the functions $U_{1} \ldots U_{5}$ are given in Appendix $\mathrm{A}$ (Eqs. (A.14) etc.).

For the case $n=-2$ the final exponential term in Eq. (15) can be neglected which has an effect of $<5 \%$ for $T \leq 5$ up to halo masses of $10^{16} M_{\odot}$. Note that Eq. (15) without the exponential term can also be obtained by asymptotic expansion of Eq. (10), which is important for generalizing the present discussion to other power spectra. Solving the truncated equation for $R$ yields the radius-mass relation

$R(M)=\frac{S_{1} S_{2}^{1 / 3}+S_{3}-S_{4} S_{2}^{-1 / 3}}{S_{5}}$

where the functions $S_{1} \ldots S_{5}$ are given in Appendix A (Eqs. (A.29) etc.).

\section{Halo mass function}

We define the halo mass function $n(M)=f(M) \bar{\rho} / M$ as the number of dark matter halos per unit volume and mass. As first shown in BCEK, the fraction $f(M)$ may be obtained from the first passage time distribution function of the assumed diffusion process.

Let $\tau$ be a random variable which determines the first passage time when $\delta(t)$ achieves the critical value $\delta_{\mathrm{c}}$. Taking into account that $\delta(t) \in \mathcal{N}\left(-\beta \sigma^{2}(t), \sigma^{2}(t)\right)$, let us define the process $\Delta(t)$ obtained by replacing the origin to $2 \delta_{\mathrm{c}}$ such that $\Delta(t) \in \mathcal{N}\left(2 \delta_{\mathrm{c}}-\beta \sigma^{2}(t), \sigma^{2}(t)\right)$. Both processes are Gaussian. Calculating the probabilities $p(t)$ and $P(t)$ of the trajectories of $\delta(t)$ and $\Delta(t)$ which attain the level $\delta_{\mathrm{c}}$ at the same moment $t$, we obtain

$p(t)=P(t) \exp \left(-2 \delta_{\mathrm{c}} \beta\right)$. 

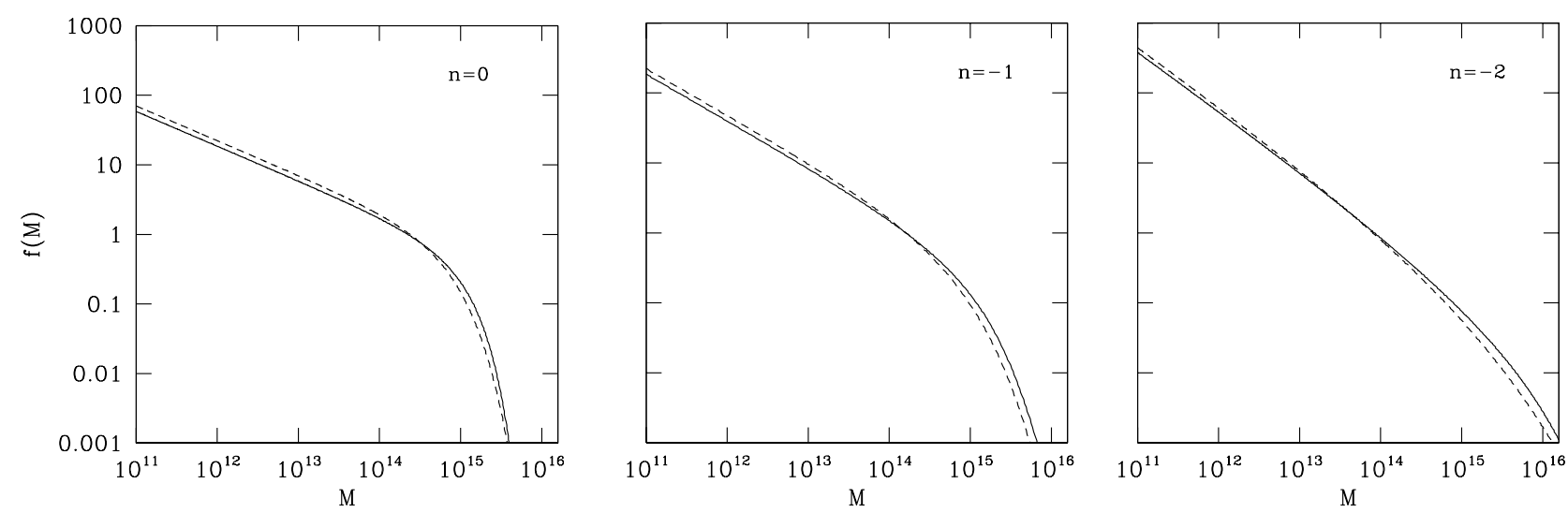

Fig. 3. Mass functions $f(M)$ for Gaussian fluctuation fields with a power spectrum with $n=0,-1,-2$. Mass $M$ in solar units: $T=0, \beta=0$ (dashed lines), and $T=0.23, \beta=0$ (continuous lines). The computations assume the normalized cosmic mass density $\Omega_{m}=0.27$, the standard normalization $\sigma_{8}=0.9$ and the normalized Hubble constant $h=0.7$.

The set of trajectories of $\delta(s), 0 \leq s \leq t$, with asymptotic end points $\delta(t) \in\left(-\infty, \delta_{\mathrm{c}}\right)$ which attain the level $\delta_{\mathrm{c}}$ at the time $\tau, \tau<$ $t$ can be associated with the set of trajectories of $\Delta(s), 0 \leq s \leq$ $t$, which have the asymptotic end points $\Delta(t) \in\left(-\infty, \delta_{\mathrm{c}}\right)$. In fact, by means of the inclusion $\Delta(t) \in\left(-\infty, \delta_{\mathrm{c}}\right)$ it follows that there exists a time $\tau, \tau<t$, such that $\Delta(t)=\delta_{\mathrm{c}}$. Equation (3) implies that $\mathrm{d} \delta(t)=V(t) \mathrm{d} t-\beta \mathrm{d} \sigma^{2}(t)$, where $V(t)=\frac{1}{T} \int_{0}^{t} \mathrm{e}^{-\frac{t-s}{T}} \mathrm{~d} W(s)$ is the Ornstein-Uhlenbeck process. Hence, increments of the processes $\delta(t)$ and $\Delta(t)$ are driven by the Markov process $V(t)$. This means that given a trajectory of $\delta(t)$, which goes through the point $\delta_{\mathrm{c}}$ at the moment $t=\tau$, there exists the trajectory of $\Delta(t)$ which coincides with the trajectory of $\delta(t)$ for $t \geq \tau$. The Markov property of $V(t)$ guarantees a possibility to construct this trajectory. It is thus sufficient to give $\Delta(t)$ the same values of the driving Ornstein-Uhlenbeck process $V(t)$ as $\delta(t)$ for $t \geq \tau$. On the other hand, the probability of trajectories $\delta(t)$ and $\Delta(t)$ which achieve the level $\delta_{\mathrm{c}}$ at the same moment $\tau$ are connected by Eq. (20). Hence, the relation (20) implies

$P\left(\delta(t) \leq \delta_{\mathrm{c}} \mid \delta(\tau)=\delta_{\mathrm{c}}, \tau<t\right)=\mathrm{e}^{-2 \delta_{\mathrm{c}} \beta} P\left(\Delta(t) \leq \delta_{\mathrm{c}}\right)$,

so that

$$
\begin{aligned}
F(t)= & P(\tau \geq t)=P\left(\delta(t) \leq \delta_{\mathrm{c}}\right)-P\left(\delta(t) \leq \delta_{\mathrm{c}} \mid \tau<t\right) \\
= & \frac{1}{\sqrt{2 \pi \sigma^{2}(t)}}\left\{\int_{-\infty}^{\delta_{\mathrm{c}}} \exp \left[-\frac{\left(x+\beta \sigma^{2}(t)\right)^{2}}{2 \sigma^{2}(t)}\right] \mathrm{d} x\right. \\
& \left.-\exp \left(-2 \delta_{\mathrm{c}} \beta\right) \int_{-\infty}^{\delta_{\mathrm{c}}} \exp \left[-\frac{\left(x-2 \delta_{\mathrm{c}}+\beta \sigma^{2}(t)\right)^{2}}{2 \sigma^{2}(t)}\right] \mathrm{d} x\right\} .
\end{aligned}
$$

For the normalized loss rate of trajectories, $f(t)=-\frac{\mathrm{d} F(t)}{\mathrm{d} t}$, we obtain the density

$$
\begin{aligned}
f(t) \mathrm{d} t & =\frac{1}{\sqrt{2 \pi}} \frac{\delta_{\mathrm{c}}}{\sigma^{3}(t)} \exp \left\{-\frac{\left[\delta_{\mathrm{c}}+\beta \sigma^{2}(t)\right]^{2}}{2 \sigma^{2}(t)}\right\} \mathrm{d} \sigma^{2}, \\
& =f\left(\sigma^{2}\right) \mathrm{d} \sigma^{2} .
\end{aligned}
$$

Written in this form, the theoretical mass function shows no dependency on $T$ and reproduces for $\beta=0$ the prediction of the Markovian excursion set model. However, $\sigma^{2}(t)$, which is basically a function of mass, depends on $T$ (see Eq. (5)) so that the non-Markovianity becomes apparent after the transformation of Eq. (22) into

$f(M)=f\left(\sigma^{2}(M)\right)\left|\frac{\mathrm{d} \sigma^{2}(M)}{\mathrm{d} M}\right|$.

Unfortunately, the final formulae of the form

$f(M)=f\left(\sigma^{2}(R(M))\right)\left|\frac{\mathrm{d} \sigma^{2}(R)}{\mathrm{d} R}\right|\left|\frac{\mathrm{d} R(M)}{\mathrm{d} M}\right|$

become quite clumsy after the insertion of all relevant equations (see Appendix A).

\section{Discussion of results}

The process (Eq. (4)) corresponds to a smooth filter profile and allows a simple discussion of non-Markovian effects within the excursion set model. The model includes the standard excursion set result as a limiting case, and allows non-Markovian effects to be increased gradually by the new filter parameter $T$.

\subsection{Effects of non-Markovianity}

Figure 3 shows halo mass functions $f(M)$ for different power spectra and values of the $T$ parameter. In all cases the nonMarkovian effects described by Eq. (4) increase (decrease) the number density of high (low) mass halos. At high masses the ellipsoidality slightly decreases the number density of high mass halos and thus partially compensates non-Markovianity. At small masses both non-Markovianity and ellipsoidality decrease the number density (Fig. 4).

The result of equating the mass and the process variances in Eq. (7) is a mass filter with a profile which depends on the power spectrum of the mass distribution and thus on the mean profile of density peaks. To show that this is a generic property 


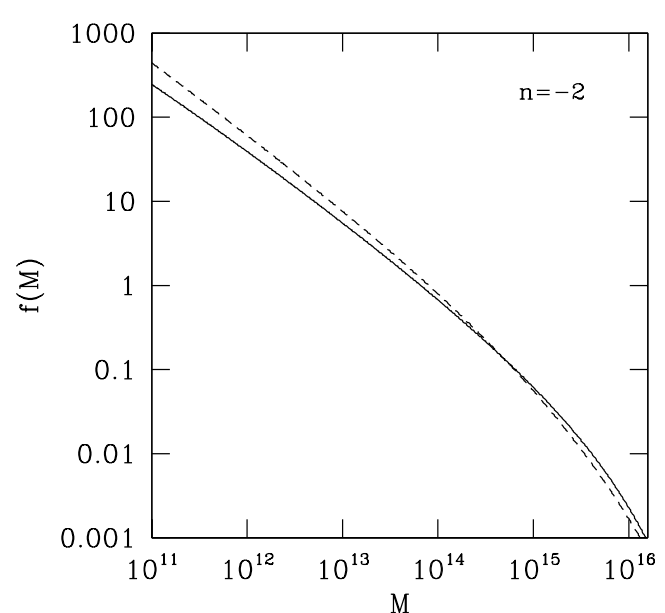

Fig. 4. Mass functions $f(M)$ for Gaussian fluctuation fields with a power spectrum with $n=-2.0$. Mass $M$ in solar units. $T=0, \beta=0$ (dashed line), and $T=0.23, \beta=0.12$ (continuous line). Further normalizations as in Fig. 3.

of non-Markovian processes with increments determined by integrals of the form (Eq. (1)), we generalize the $\left[1-\mathrm{e}^{-(t-s) / T}\right]$ term in Eq. (4) by a function $K_{T}(s, t)$ so that Eq. (7) reads

$t=\int_{0}^{t} \mathrm{~d} s K_{T}^{2}(s, t)=\int_{0}^{\infty} \frac{4 \pi k^{2} \mathrm{~d} k P(k)}{(2 \pi)^{2}}\left|W_{T}(k R)\right|^{2}$.

Moreover, from the derivation which leads to Eq. (9) we found that the resolution variable $t$ is the same for the Brownian process and our non-Markovian process. The same resolution variable was also obtained for the Ornstein-Uhlenbeck process (Schuecker et al. 2001a). We thus generalize Eq. (9) to a universal resolution variable

$t=\int_{0}^{t} \mathrm{~d} s=\int_{0}^{1 / R} \frac{4 \pi k^{2} \mathrm{~d} k P(k)}{(2 \pi)^{3}}=s[R, P(k)]$.

The combination of Eqs. (24) and (25) then suggests the equivalence $K_{T}(R, P(k))=W_{T}(k R)$ for $k R \leq 1$ and zero elsewhere. This shows that the kernel of Eq. (3) which determines the increments of the process is closely related to the profile of the mass filter.

We further conclude that for merging and accretion processes described by the comparatively simple integration scheme (Eq. (3)), the mass filter and thus how much mass is swept in from the surrounding mass by a collapsing region must also depend on the global mass distribution, and thus on the power spectrum.

Equations (10)-(19) follow a "natural" choice of the relation between the filter radius, used in the process of halo detection, and the halo mass as determined by the material which eventually collapses to form a virialized structure. However, other choices are also possible (BCEK) and still a matter of debate.

We further note that the application of filters to all spatial points of a density field, as proposed in all PS-like models, leads to a large scatter between filter mass and group mass. The introduction of non-Markovianity follows the same local filter-

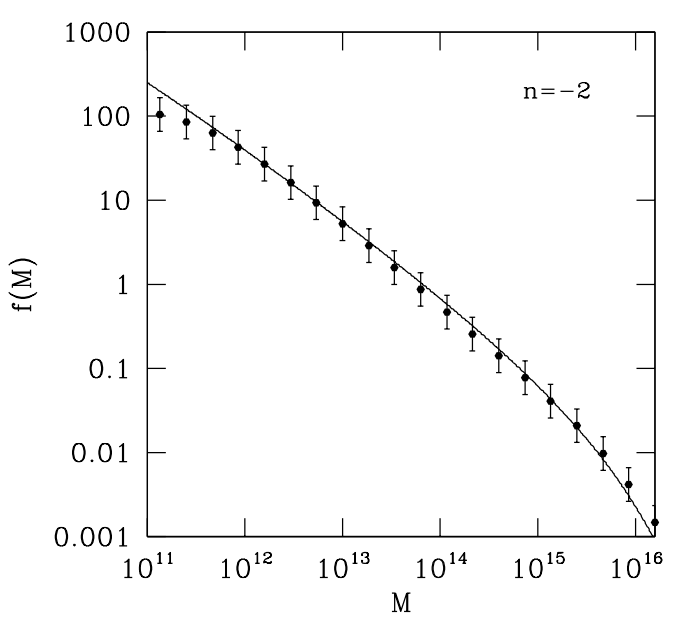

Fig. 5. Best fit mass function $f(M)$ with $T=0.23, \beta=0.12$ (continuous line) and the transformed mass function of Jenkins et al. (2001) with $20 \%$ Gaussian random errors (dots with error bars). Normalizations as in Fig. 3.

ing scheme and is thus not expected to significantly reduce the scatter on the halo-by-halo level.

\subsection{Comparison with simulated mass functions}

On the statistical level, PS-like models predict mass functions, merger rates, formation times, biasing schemes etc. remarkably accurately. A comparison of the theoretical mass functions (Eq. (23)) with the results from large and high-resolution $N$-body simulations (e.g., Jenkins et al. 2001) should thus be more fruitful and should give more important information about the significance of non-Markovian effects.

The Jenkins et al. (2001) mass function can be used for fluctuation fields with effective power spectrum slopes between $n_{\text {eff }}=-2.5$ and -1 at $\sigma=0.5$ in the mass range $10^{11}-10^{16} M_{\odot}$. Observations suggest a slope of about $n=-2.0$ (see, e.g., Schuecker et al. $2001 \mathrm{~b}$ who found $n=-1.8$ on scales $<100 h^{-1} \mathrm{Mpc}$ for X-ray clusters of galaxies - quite consistent with the power spectrum of galaxies, see their Fig. 16). We thus compare with our $n=-2$ model.

To be consistent with our "natural" choice of the radiusmass relation (Eq. (19)), the Jenkins et al. mass function (e.g. their Eq. (9)) has to be transformed accordingly by using the $\sigma^{2}(R(M))$ relation of the non-Markovian process for $n=-2$. To say it in another way, the non-Markovian effects of the process (Eq. (3)) can only become apparent after the transformation of $f\left(\sigma^{2}(R)\right)$ to $f(M)$ because non-Markovianity changes the radius-mass assignment scheme. Therefore, when we want to search for non-Markovian effects we have to transform $f\left(\sigma^{2}\right)$ of both the theoretical and the simulated mass function and test different values of $T$. For this test we multiply the prefactor $16 \pi^{2}$ in the normalization (Eq. (16)) by 1.234 to be consistent with the standard $\sigma_{8}$ normalization (obtained with the top-hat filter) for $T=0$.

The comparison with the Jenkins et al. (2001) mass function within the mass range $10^{12}-10^{16} M_{\odot}$ (for the lower limit 

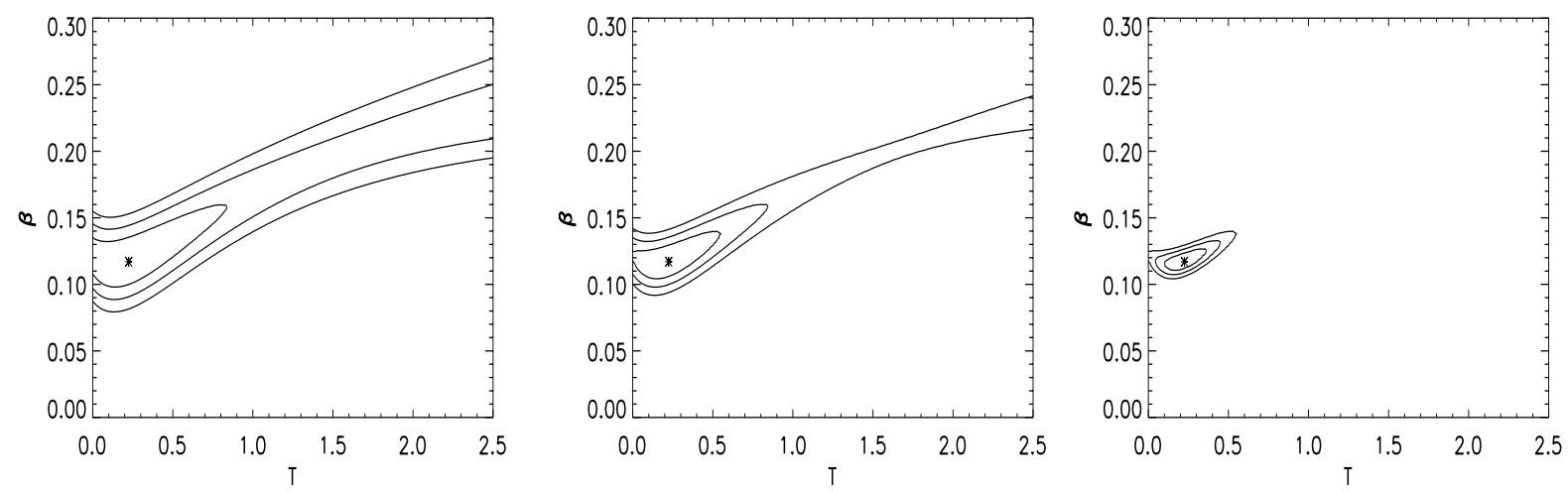

Fig. 6. $\chi^{2}$ distribution (1-3 $\sigma$ contours for three parameters) for the spectral index $n=-2$ obtained from the comparison of mass functions $f(M)$ with different $T$ and $\beta$ parameter values and the transformed Jenkins et al. (2001) mass function. Normalizations as in Fig. 3. The $\chi^{2}$ test assumes errors of $30 \%$ (left), $20 \%$ (middle), $10 \%$ (right) of the Jenkins et al. mass function.

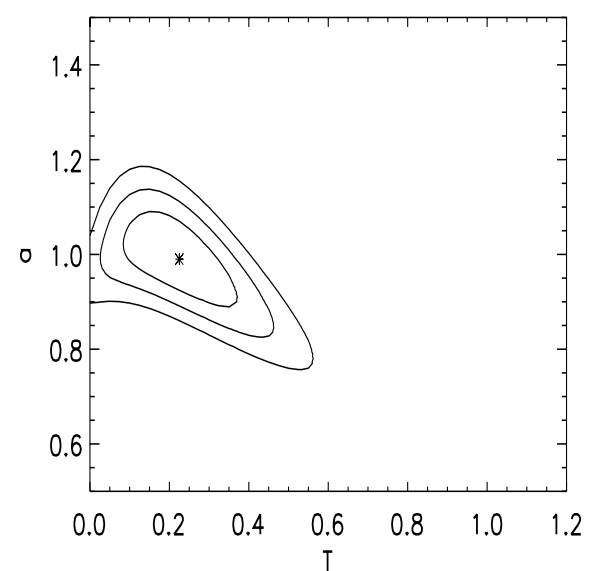

Fig. 7. $\chi^{2}$ distribution (1-3o contours for three parameters) in the $\beta=$ 0.12 plane, assuming $10 \%$ errors of the Jenkins et al. (2001) mass function.

see Sect. 6.3) gives the best fit parameter values $T=0.23$ and $\beta=0.12$ with $\chi^{2}=1.4$ for three degrees of freedom (Fig. 5). The statistical significance of the non-Markovian effects strongly depends on the assumed error of the Jenkins et al. mass function. For errors of 20-30\%, non-Markovian effects must be regarded as insignificant, whereas for $10 \%$ errors, non-Markovianity is clearly detected (Fig. 6). We thus conclude that for the most optimistic error estimates of the Jenkins et al. mass function, its shape suggests the presence of nonMarkovian effects, i.e., effects of the environment on the coherence of the collapse. Moreover, non-Markovian effects seem to be not very large and cosmic mass functions with errors better than $10 \%$ are needed for their clear detection.

It should be mentioned that we simultaneously tested in Fig. 6 for the significance of the scaling parameter $a$ introduced by SMT to account for a failure of the simple counting argument of the excursion set formalism (R. Sheth, private communication). The best fit has $a=0.99$ (Fig. 7) which means that the inclusion of non-Markovian effects does not require this correction. Therefore, the likelihood contours in Fig. 6 are only plotted in the $a=1$ plane.

\subsection{Effects of ellipsoidality}

The effects of ellipsoidality on the halo mass function are in general much larger and are detected for all random errors $\leq 30 \%$ with clear significance. The best fit $\beta=0.12$ is lower than $\beta=0.3$ obtained from simulations (see Sect. 2). In addition, the model starts to deviate from the Jenkins et al. function at masses smaller than $10^{12} M_{\odot}$. In order to understand these failures of the model one should not forget that our goal to give a full analytic treatment of the problem forces us to approximate the ellipsoidal collapse by a linear drift. Sheth \& Tormen (2002) give a simple prescription for approximating the solution to the general barrier in the Markov case which better fits the low-mass range. Therefore, a useful direction for future work is to see if their method works in our particular non-Markovian context also.

Future observed mass functions from e.g. X-ray clusters of galaxies and lensing studies have high enough precision for detailed studies of non-Markovian effects expected in the highmass regime. On the theoretical side, further studies are in preparation to predict mass functions also for Cold Dark Matter power spectra to improve the comparison with observed and simulated mass functions.

Acknowledgements. We would like to thank Hans Böhringer, Gerard Lemson, Gabriel Pratt, Ravi Sheth, and the anonymous referee for helpful comments on the manuscript. GGA is partially supported by INTAS grant No. 00-738 and PS by DLR grant No. 50 OR 0108.

\section{References}

Bond, J. R., Cole, S., Efstathiou, G., \& Kaiser, N. 1991, ApJ, 379, 440 (BCEK)

Bond, J. R., \& Myers, S. T. 1996, ApJS, 103, 1

Cavaliere, A., \& Menci, N. 1994, ApJ, 435, 528

Corless, R. M., Gonnet, G. H., Hare, D. E. G., Jeffrey, D. J., \& Knuth, D. E. 1996, Adv. Comput. Math. 5, 329

Jenkins, A., Frenk, C. S., White, S. D. M., et al. 2001, MNRAS, 321, 372 
Feller, W. 1968, An introduction to probability theory and its applications, I, Third edition (New York-London-Sydney: John Wiley \& Sons, Inc.)

Lacey, C., \& Cole, S. 1993, MNRAS, 262, 627

Lee, J., \& Shandarin, S. F. 1998, ApJ, 500, 14

Peebles, P. J. E. 1980, The Large-Scale Structure of the Universe (Princeton, New Jersey: Princeton Univ. Press)

Lucchin, F., \& Matarrese, S. 1988, ApJ, 330, 535

Monaco, P. 1995, ApJ, 447, 23

Press, W. H., \& Schechter, P. 1974, ApJ, 187, 425

Schuecker, P., Böhringer, H., Arzner, K., \& Reiprich, T. H. 2001a, A\&A, 370, 715
Schuecker, P., Böhringer, H., Guzzo, L., et al. 2001b, A\&A, 368, 86

Sheth, R. K., Mo, H. J., \& Tormen, G. 2001, MNRAS, 323, 1 (SMT)

Sheth, R. K., \& Tormen, G. 2002, MNRAS, 329, 61

Valageas, P., \& Schaeffer, R. 1997, A\&A, 328, 435

White, S. D. M. 1996, in Cosmology and Large Scale Structure, Les Houches Session LX, ed. R. Schaeffer, J. Silk, M. Spiro, \& J. Zinn-Justin (Amsterdam: Elsevier), 349

White, S. D. M. 1997, in The Evolution of the Universe, Dahlem Workshop Reports, ed. G. Börner, \& S. Gottlöber (Chichester: John Wiley \& Sons), 227

White, S. D. M., \& Rees, M. 1978, MNRAS, 183, 341

Zel'dovich, Ya. B. 1970, A\&A, 5, 20 
G. Amosov and P. Schuecker: Non-Markov excursion set model, Online Material p 1

\section{Online Material}




\section{Appendix A: Computation of mass functions}

A summary is given of the equations used for the computation of the dark matter halo mass functions discussed in the main text. The meaning of the different symbols is as follows.

$f\left(\sigma^{2}\right)$ dark matter mass function as a function of the variance $\sigma^{2}$ of the random mass field or of the diffusion process. $f(M)$ dark matter halo mass function as a function of mass $M$. $\delta_{\mathrm{c}}$ critical density threshold. $\beta$ parameter regulating the ellipsoidality of the collapse ( $\beta=0$ spherical collapse). $T$ parameter regulating the coherence of the collapse, i.e., the deviation from Markovianity ( $T=0$ traditional Markovian excursion set model). $P_{0}$ amplitude of the scale-invariant power spectrum in $P(k)=P_{0} k^{n}$ with the spectral index $n . \bar{\rho}$ mean cosmic matter density. $\Omega_{m}$ normalized cosmic matter density. $h$ Hubble constant in units of $100 \mathrm{~km} \mathrm{~s}^{-1} \mathrm{Mpc}^{-1} . t$ pseudo diffusion time or mass resolution variable. $R$ filter radius. $W(z)$ the principal branch of the Lambert W-function. $U_{1} \ldots U_{5}, S_{1} \ldots S_{5}$ auxiliary functions, and $U_{1}^{\prime} \ldots U_{5}^{\prime}, S_{1}^{\prime} \ldots S 5$ their derivatives with respect to $M$.

The mass functions $f\left(\sigma^{2}\right)$ with the spectral indices $n=$ $0,-1,-2$ have the same form

$f\left(\sigma^{2}\right)=\frac{1}{\sqrt{2 \pi}} \frac{\delta_{\mathrm{c}}}{\sigma^{2}} \mathrm{e}^{-\frac{\left|\delta_{\mathrm{c}}+\beta \sigma^{2}\right|^{2}}{2 \sigma^{2}}}$,

and the same $\sigma^{2}(t)$ dependence

$\sigma^{2}=t-\frac{3}{2} T+2 T \mathrm{e}^{-t / T}-\frac{T}{2} \mathrm{e}^{-2 t / T}$.

Moreover, the cosmic mass density

$\bar{\rho}=2.7755 \Omega_{m} h^{2} 10^{11} M_{\odot} \mathrm{Mpc}^{-3}$

is the same for the power spectra.

\section{A.1. Mass function for a power spectrum with the spectral index $n=0$}

Dark matter halo mass function:

$f(M)=f\left(\sigma^{2}\left(R^{3}(M)\right)\right)\left|\frac{\mathrm{d} \sigma^{2}\left(R^{3}\right)}{\mathrm{d} R^{3}}\right|\left|\frac{\mathrm{d} R^{3}(M)}{\mathrm{d} M}\right|$.

Pseudo diffusion time or mass resolution variable:

$t=\frac{P_{0}}{6 \pi^{2} R^{3}}$.

Filter radius-mass relation:

$R^{3}=\frac{M}{6 \pi^{2} \bar{\rho}+\frac{6 \pi^{2} T M}{P_{0}}[1+W(z)]}$.

Normalization of the matter power spectrum:

$P_{0}=3072 \pi^{2}\left\{\sigma_{8}^{2}+1.98 T\left[1-\mathrm{e}^{-\left(\sigma_{8}^{2} / T\right)^{0.363}}\right]\right\}$.

Argument of the Lambert $W$ function:

$z=-\exp \left[-\left(1+\frac{\bar{\rho} P_{0}}{M T}\right)\right]$
Gradient of filter radius with mass:

$$
\begin{aligned}
\frac{\mathrm{d} R^{3}}{\mathrm{~d} M}= & \frac{1}{6 \pi^{2} \bar{\rho}+\frac{6 \pi^{2} T M}{P_{0}}[1+W(z)]} \\
& -\frac{P_{0} M\left\{T[1+W(z)]+\frac{\bar{\rho} P_{0}}{e M} \frac{W(z)}{1+W(z)}\right\}}{6 \pi^{2}\left\{\bar{\rho} P_{0}+T M[1+W(z)]\right\}^{2}} .
\end{aligned}
$$

Gradient of mass variance with filter radius:

$\frac{\mathrm{d} \sigma^{2}\left(R^{3}\right)}{\mathrm{d} R^{3}}=-\frac{P_{0}}{6 \pi^{2} R^{6}}\left(1-2 \mathrm{e}^{-\frac{P_{0}}{6 \pi^{2} R^{3} T}}+\mathrm{e}^{-\frac{P_{0}}{3 \pi^{2} R^{3} T}}\right)$.

More information about the Lambert $W$ function can be found in Corless et al. (1996).

\section{A.2. Mass function for a power spectrum with the spectral index $n=-1$}

Dark matter halo mass function:

$f(M)=f\left(\sigma^{2}(R(M))\right)\left|\frac{\mathrm{d} \sigma^{2}(R)}{\mathrm{d} R}\right|\left|\frac{\mathrm{d} R(M)}{\mathrm{d} M}\right|$.

Pseudo diffusion time or mass resolution variable:

$t=\frac{P_{0}}{4 \pi^{2} R^{2}}$

Filter radius-mass relation:

$R(M)=\frac{\left(U_{1}+3 \sqrt{U_{2}}\right)^{1 / 3}}{U_{3}}+\frac{U_{4}}{\left(U_{1}+3 \sqrt{U_{2}}\right)^{1 / 3}}-U_{5}$.

Auxiliary functions for the computation of radius-mass relation:

$U_{1}=9 \pi^{4} \bar{\rho}^{2} P_{0}^{3} M-4 \pi^{6} M^{3} T^{3}$

$U_{2}=9 \pi^{8} \bar{\rho}^{4} P_{0}^{6} M^{2}-8 \pi^{10} \bar{\rho}^{2} P_{0}^{3} M^{4} T^{3}$

$U_{3}=3 \cdot 2^{2 / 3} \pi^{2} \bar{\rho} P_{0}$

$U_{4}=\frac{2^{2 / 3} \pi^{2} M^{2} T^{2}}{3 \bar{\rho} P_{0}}$.

$U_{5}=\frac{M T}{3 \bar{\rho} P_{0}}$

Normalization of the matter power spectrum:

$P_{0}=256 \pi^{2}\left[\sigma_{8}^{2}+1.98 T\left(1-\mathrm{e}^{-\left(\frac{\sigma_{8}^{2}}{T}\right)^{0.363}}\right)\right] \mathrm{Mpc}^{2}$.

Gradient of filter radius with mass:

$$
\begin{aligned}
\frac{\mathrm{d} R(M)}{\mathrm{d} M} & =\frac{U_{1}^{\prime}+\frac{3}{2} U_{2}^{-1 / 2} U_{2}^{\prime}}{3 U_{3}\left(U_{1}+3 \sqrt{U_{2}}\right)^{2 / 3}}+\frac{U_{4}^{\prime}}{\left(U_{1}+3 \sqrt{U_{2}}\right)^{1 / 3}} \\
& -\frac{U_{4}\left(U_{1}^{\prime}+\frac{3}{2} U_{2}^{-1 / 2} U_{2}^{\prime}\right)}{3\left(U_{1}+3 \sqrt{U_{2}}\right)^{4 / 3}}-U_{5}^{\prime} .
\end{aligned}
$$


G. Amosov and P. Schuecker: Non-Markov excursion set model, Online Material p 3

Derivatives of auxiliary functions with respect to mass:

$U_{1}^{\prime}=9 \pi^{4} \bar{\rho}^{2} P_{0}^{3}-12 \pi^{6} M^{2} T^{3}$

$U_{2}^{\prime}=18 \pi^{8} \bar{\rho}^{4} P_{0}^{6} M-32 \pi^{10} \bar{\rho}^{2} P_{0}^{3} M^{3} T^{3}$

$U_{4}^{\prime}=\frac{2^{5 / 3} \pi^{2} M T^{2}}{3 \bar{\rho} P_{0}}$

$U_{5}^{\prime}=\frac{T}{3 \bar{\rho} P_{0}}$.

Gradient of mass variance with filter radius:

$\frac{\mathrm{d} \sigma^{2}(R)}{\mathrm{d} R}=-\frac{P_{0}}{2 \pi^{2} R^{3}}\left(1-2 \mathrm{e}^{-\frac{P_{0}}{4 \pi^{2} R^{2} T}}+\mathrm{e}^{-\frac{P_{0}}{2 \pi^{2} R^{2} T}}\right)$.

The determination of the radius-mass relation neglects the exponential term in Eq. (13).

\section{A.3. Mass function for a power spectrum with the spectral index $n=-2$}

Dark matter halo mass function:

$f(M)=f\left(\sigma^{2}(R(M))\right)\left|\frac{\mathrm{d} \sigma^{2}(R)}{\mathrm{d} R}\right|\left|\frac{\mathrm{d} R(M)}{\mathrm{d} M}\right|$.

Pseudo diffusion time or mass resolution variable:

$t=\frac{P_{0}}{2 \pi^{2} R}$.

Filter radius-mass relation:

$R(M)=\frac{S_{1} S_{2}^{1 / 3}+S_{3}-S_{4} S_{2}^{-1 / 3}}{S_{5}}$.

Auxiliary functions for the computation of the radius-mass relation:

$$
\begin{aligned}
S_{1}= & 6\left(2 \pi^{4}\right)^{1 / 3} \\
S_{2}= & P_{0}^{3} M\left[9 \bar{\rho}^{2} P_{0}^{6}+72 \pi^{4} \bar{\rho} P_{0}^{3} M T^{3}\right. \\
& +256 \pi^{8} M^{2} T^{6}+3\left(\bar{\rho} P_{0}^{3}+8 \pi^{4} M T^{3}\right) \\
& \left.\times \sqrt{9 \bar{\rho}^{2} P_{0}^{6}+48 \pi^{4} \bar{\rho} P_{0}^{3} M T^{3}+128 \pi^{8} M^{2} T^{6}}\right]
\end{aligned}
$$

$$
\begin{aligned}
& S_{3}=48 \pi^{4} P_{0} M T^{2} \\
& S_{4}=12 \cdot 2^{2 / 3} \pi^{8 / 3} P_{0}^{2} M T\left(3 \bar{\rho} P_{0}^{3}+8 \pi^{4} M T^{3}\right) \\
& S_{5}=36\left(\pi^{2} \bar{\rho} P_{0}^{3}+8 \pi^{6} M T^{3}\right) .
\end{aligned}
$$

Normalization of the matter power spectrum:

$P_{0}=16 \pi^{2}\left[\sigma_{8}^{2}+1.98 T\left(1-\mathrm{e}^{-\left(\frac{\sigma_{8}^{2}}{T}\right)^{0.363}}\right)\right] \mathrm{Mpc}$

Gradient of filter radius with mass:

$$
\begin{aligned}
\frac{\mathrm{d} R(M)}{\mathrm{d} M}= & \frac{\frac{1}{3} S_{1} S_{2}^{-2 / 3} S_{2}^{\prime}+S_{3}^{\prime}-S_{4}^{\prime} S_{2}^{-1 / 3}+\frac{1}{3} S_{4} S_{2}^{-4 / 3} S_{2}^{\prime}}{S_{5}} \\
& -\frac{\left(S_{1} S_{2}^{1 / 3}+S_{3}-S_{4} S_{2}^{-1 / 3}\right) S_{5}^{\prime}}{S_{5}^{2}}
\end{aligned}
$$

Derivatives of auxiliary functions with respect to mass:

$$
\begin{aligned}
S_{2}^{\prime}= & 9 \bar{\rho}^{2} P_{0}^{9}+144 \pi^{4} \bar{\rho} P_{0}^{6} T^{3} M+768 \pi^{8} P_{0}^{3} T^{6} M^{2} \\
& +\left(3 \bar{\rho} P_{0}^{6}+48 \pi^{4} P_{0}^{3} T^{3} M\right) \\
& \times \sqrt{9 \bar{\rho}^{2} P_{0}^{6}+48 \pi^{4} \bar{\rho} P_{0}^{3} M T^{3}+128 \pi^{8} M^{2} T^{6}} \\
& +\frac{\left(3 \bar{\rho} P_{0}^{6} M+24 \pi^{4} P_{0}^{3} T^{3} M^{2}\right)\left(48 \pi^{4} \bar{\rho} P_{0}^{3} T^{3}+256 \pi^{8} M T^{6}\right)}{2 \sqrt{9 \bar{\rho}^{2} P_{0}^{6}+48 \pi^{4} \bar{\rho} P_{0}^{3} M T^{3}+128 \pi^{8} M^{2} T^{6}}} \\
S_{3}^{\prime}= & 48 \pi^{4} P_{0} T^{2} \\
S_{4}^{\prime}= & 12 \cdot 2^{2 / 3} \pi^{8 / 3} P_{0}^{2} T\left(3 \bar{\rho} P_{0}^{3}+16 \pi^{4} M T^{3}\right) \\
S_{5}^{\prime}= & 288 \pi^{6} T^{3}
\end{aligned}
$$

Gradient of mass variance with filter radius:

$\frac{\mathrm{d} \sigma^{2}(R)}{\mathrm{d} R}=-\frac{P_{0}}{2 \pi^{2} R^{2}}\left(1-2 \mathrm{e}^{-\frac{P_{0}}{2 \pi^{2} R T}}+\mathrm{e}^{-\frac{P_{0}}{\pi^{2} R T}}\right)$.

The determination of the radius-mass relation neglects the exponential term in Eq. (15). 